\title{
Human pluripotent stem cells: genetic instability or stability?
}

\author{
Peter W Andrews*,1 \\ ${ }^{1}$ Department of Biomedical Science, Centre for Stem Cell Biology, The University of Sheffield, Sheffield S10 2TN, United Kingdom \\ *Author for correspondence: P.W.Andrews@sheffield.ac.uk
}

“'Despite their propensity for acquiring recurrent genomic changes, PSCs are not inherently genetically unstable, though the mechanisms that they deploy to maintain genomic fidelity differ from somatic cells."

First draft submitted: 21 January 2021; Accepted for publication: 9 February 2021; Published online: 2 March 2021

Keywords: genetic $\bullet$ human $\bullet$ karyotype $\bullet$ mutations $\bullet$ pluripotent stem cells

Unsurprisingly, human pluripotent stem cells (PSCs), whether embryonic stem cells or induced pluripotent stem cells, are subject to continual genetic change and evolution during periods of extended culture. Perhaps more surprising, however, is the speed with which novel genetic variants can become predominant in a culture, sometimes within four to five passages [1]. These rapidly appearing variants are often recurrent, repeatedly affecting the same regions of the genome. Although, most of the genome may be affected by mutation, more commonly these repetitive variants involve gains of regions of the long arms of chromosomes 1, 17 and 20 or the short arm of chromosome 12 or losses of regions on chromosomes 10 and 18 [2,3]. By contrast a few regions of the genome, notably chromosome 4, seem to remain remarkably stable, with few variants ever reported. Much of the information about such variants has come from karyotype analysis, both by G-banding or genomic array methods, but recently whole genome sequencing has also revealed repetitive mutations in single gene, TP53, that is not typically affected by the karyotypic changes that are commonly seen [4].

Although differential susceptibility to mutation across the genome may contribute, it seems most likely that the repetitive nature of the common genetic variants of human PSCs reflects selection of randomly occurring mutants that happen to offer cells enhanced proliferation or survival compared with their wild-type parents. It is often possible to observe the progressive takeover of a culture by a novel variant during successive passages or, similarly, if wild-type cultures are spiked with an established variant [5]. Further, in the case of the long arm of chromosome 20, which is frequently subject to genomic gains, analysis of genes located in a minimal commonly amplified region identified $B C L 2 L 1$, a regulator of apoptosis, as the driver gene that provides cells that have acquired this amplification with a growth advantage [6,7]. The anti-apoptotic protein, BCL2-XL, encoded by BCL2L1, is expressed in PSCs and transfection of PSCs with a vector encoding BCL2-XL also provides the cells with a growth advantage over their wild-type parental cells. The dominant negative mutations seen in TP53 could similarly provide a survival advantage by limiting apoptosis. However, driver genes for the other common recurrent changes remain to be identified, although candidates have been suggested, such as MDM4 on chromosome 1q, BIRC5 on chromosome 17q or NANOG on chromosome 12p [3].

The tendency of PSCs to acquire genetic variants is a significant concern for their various applications. If not recognized, acquired variants might interfere with studies that use these cells for disease modeling or drug screening. More critically, they could also pose substantial safety risks if present in cells used for regenerative medicine, especially since a number of the variants involve genes such as TP53 or BCL2L1 that are also well known to be associated with cancer. Nevertheless, the consequences of particular mutations in PSCs for particular applications is often difficult to evaluate. The development of cancers typically involves multiple mutations that accumulate over time. A single cancer associated genetic change may not be enough to be tumorigenic alone, though no doubt it will increase the possibility that a cell could acquire enough mutations over time to become malignant. From this perspective 
the risk associated with a single genetic variant maybe context dependent (e.g., it might be considered a more serious concern for treatments of younger than for older patients), but there is currently no consensus about how to assess that risk. However, the situation is more complex. In the case of genetic variants that involve a copy number variation (CNV), it is not sufficient to consider just the effects of the driver gene but also the effects of the many other linked 'hitch-hiker' genes encoded in the CNV. Also, although much attention has focused on the potential of undifferentiated PSCs to produce malignant teratocarcinomas, these cells would only be introduced into a patient as contaminants of their differentiated derivatives transplanted to effect a tissue repair. A more important concern is whether the differentiated cells required for a treatment themselves could acquire malignant properties and how these might be affected by the genetic variants.

The obvious solution to the acquisition of genetic variants should be to avoid the use of such cells, but in practice this is seriously hampered by the low sensitivity of the available tools for detecting such variants, particularly karyotypic changes and CNVs, a difficulty that is often poorly recognized [8]. For example, publications may report that the cells used in a study have a 'normal', euploid karyotype but then fail to report details such as the number of metaphases assessed, for example, by G-banding karyology. In fact, the typical clinical standard of assessing 20-30 metaphases will likely overlook the presence of up to $10-20 \%$ of variant cells, while other approaches such as array karyotyping, comparative genomic hybridization, PCR or fluorescent in situ hybridization are little better [8]. On the whole, using current methodologies, it would be possible that a preparation of cells deemed to be free of genetic variants may, in fact, easily include $10 \%$ or more variant cells.

Given the difficulties of reliably screening out lines that carry variants it is important to seek ways to minimize the appearance of variants in the first instance. Although the appearance of recurrent variants makes headlines, it is easy to overlook that the rate at which these occur is, nevertheless, low. In a screen carried out by the International Stem Cell Initiative consortium [2] of genetic variants in earlier and later passages of PSCs, 79 lines out of 120 remained karyotypically normal throughout the study, while in a whole genome screen of 140 PSCs lines, only five were found to harbor mutations in TP53 [4]. Further, in a recent direct study we found that the underlying mutation rate in human PSCs is indeed low - at least comparable, if not lower than in somatic cells and certainly lower than in various cancer cells [9]. It was also notable that oxidative stress was a key contributor to mutation and that the mutation rate could be lowered further by culture under low oxygen conditions.

Nevertheless, in apparent contradiction to evidence of a low mutation rate in PSCs, in another study we found that, much more than somatic cells, PSCs are particularly susceptible to DNA replication stress, which can lead to DNA double stranded breaks and mutation, as well as aberrant mitoses that can result in karyotypic abnormalities [10]. But PSCs also differ from somatic cells in their response to DNA replication stress. Whereas somatic cells temporarily arrest DNA replication, resuming when appropriate conditions restored, PSCs respond by entering apoptosis [11]. This may reflect the exigencies of early embryogenesis, when rapid cell proliferation is required but where an early mutation, if carried forward, could be disastrous for the embryo as a whole: sacrifice of potentially mutated cells may well be more advantageous that attempting to repair the damage. By contrast once somatic cells are incorporated into an organized tissue it may be more advantageous to repair any damage than to eliminate the cell. In any event, one mechanism for the recurrent variants observed in PSCs is that they include mutations that tend to counter the normally low apoptotic threshold of these cells. Apart from BCL2L1 in the chr20 amplicon and TP53, candidate genes associated with gains of chromosomes $1 \mathrm{q}$ and $17 \mathrm{q}$ are anti-apoptotic while a candidate gene associated with the recurrent deletion on chromosome 18, NOXA, is pro-apoptotic and highly expressed in PSCs [3].

From these observations it might be expected that attempts to improve growth of PSC cultures by inhibiting apoptosis, for example, by use of Rho Kinase inhibitors, could encourage the appearance of genetic variants by preventing the clearing of cells with DNA damage, although in our mutation rate study the Rho Kinase inhibitor, Y27632, did not affect the mutation rate itself [9]. On the other hand, our study of replication stress in PSC [10] suggested that their susceptibility may be the result of a shortened G1 associated with their rapid cell division rates, and a consequent deficiency of DNA precursors. Indeed, we found that addition of exogenous nucleosides to their culture medium does reduce DNA replication stress and evidence of DNA damage in these cells [10]. Further, it markedly reduced abnormalities at mitosis, so providing a simple method for reducing the appearance of genetic variants.

Although the acquisition of genetic change in cultured human PSCs is inevitable and poses challenges for the varied uses of these cells, the problems are certainly manageable provided that they are recognized. Despite their propensity for acquiring recurrent genomic changes, PSCs are not inherently genetically unstable, though the 
mechanisms that they deploy to maintain genomic fidelity differ from somatic cells. Certainly, their mutation rates can be lowered further by modifications to culture conditions, such as the use of low oxygen atmospheres or inclusion of nucleosides in the culture media $[9,10]$. Nevertheless, it is essential that in all studies researchers using PSCs properly monitor their cells for genetic variants, and fully report how they have done this. Accumulating that information over time is the only way in which we will be able to fully evaluate the consequences of particular changes [12].

\section{Financial \& competing interests disclosure}

The author has no relevant affiliations or financial involvement with any organization or entity with a financial interest in or financial conflict with the subject matter or materials discussed in the manuscript. This includes employment, consultancies, honoraria, stock ownership or options, expert testimony, grants or patents received or pending, or royalties.

No writing assistance was utilized in the production of this manuscript.

\section{Open access}

This work is licensed under the Creative Commons Attribution 4.0 License. To view a copy of this license, visit http://creativecommons.org/licenses/by/4.0/

\section{References}

1. Draper JS, Smith K, Gokhale P et al. Recurrent gain of chromosomes $17 \mathrm{q}$ and 12 in cultured human embryonic stem cells. Nat. Biotechnol. 22, 53-54 (2004).

2. Amps K, Andrews PW, Anyfantis G et al. Screening ethnically diverse human embryonic stem cells identifies a chromosome 20 minimal amplicon conferring growth advantage. Nat. Biotechnol. 29, 1132-1144 (2011).

3. Halliwell J, Barbaric I, Andrews PW. Acquired genetic changes in pluripotent stem cells: origins and consequences. Nat. Rev. Mol. Cell Biol. 21, 715-728 (2020).

4. Merkle FT, Ghosh S, Kamitaki N et al. Human pluripotent stem cells recurrently acquire and expand dominant negative P53 mutations. Nature 545, 229-233 (2017).

5. Olariu V, Harrison NJ, Coca D et al. Modeling the evolution of culture-adapted human embryonic stem cells. Stem Cell Res. 4, 50-56 (2010).

6. Avery S, Hirst AJ, Baker D et al. Bcl-xL mediates the strong selective advantage of a 20q11.21 amplification commonly found in human embryonic stem cell cultures. Stem Cell Rep. 1, 379-386 (2013).

7. Nguyen HT, Geens M, Mertzanidou A et al. Gain of 20q11.21 in human embryonic stem cells improves cell survival by increased expression of Bcl-xL. Mol. Hum. Reprod. 20, 168-177 (2014).

8. Baker D, Hirst AJ, Gokhale PJ et al. Detecting genetic mosaicism in cultures of human pluripotent stem cells. Stem Cell Rep. 7 , 998-1012 (2016).

9. Thompson O, von Meyenn F, Hewitt Z et al. Low rates of acquisition of de novo mutations in human pluripotent stem cells under different culture conditions. Nat. Commun. 11, 1528 (2020).

10. Halliwell JA, Frith TJR, Laing O et al. Nucleosides rescue replication-mediated genome instability of human pluripotent stem cells. Stem Cell Rep. 14, 1009-1017 (2020b).

11. Desmarais JA, Hoffmann MJ, Bingham G, Gagou ME, Meuth M, Andrews PW. Human embryonic stem cells fail to activate CHK1 and commit to apoptosis in response to DNA replication stress. Stem Cells 30, 1385-1393 (2012).

12. Andrews PW, Ben-David U, Benvenisty N et al. Assessing the safety of human pluripotent stem cells and their derivatives for clinical applications. Stem Cell Rep. 9, 1-4 (2017). 
